\title{
QTL mapping and candidate gene analysis of peduncle vascular bundle related traits in rice by genome-wide association study
}

Laiyuan Zhai ${ }^{1}$, Tianqing Zheng ${ }^{2}$, Xinyu Wang ${ }^{1}$, Yun Wang ${ }^{2}$, Kai Chen ${ }^{2,3}$, Shu Wang ${ }^{1}$, Yun Wang ${ }^{1 *}$, Jianlong $\mathrm{Xu}^{2,3^{*}}$ (D) and Zhikang $\mathrm{Li}^{2,4}$

\begin{abstract}
Background: The vascular bundle especially in the peduncle is one of crucial limiting factors of rice yield, and it determines how plants efficiently transport photosynthetic products, mineral nutrients and water from leaf and root to panicle. However, the genetic base of rice vascular bundle related traits in the peduncle still remains unknown.

Results: The 423 panel showed substantial natural variations of peduncle vascular bundle. In total, 48 quantitative trait loci/locus (QTL) affecting the eight traits were identified throughout the genome by applying a significance threshold of $P<1.0 \times 10^{-4}$. Combined determining linkage disequilibrium (LD) blocks associated with significant SNPs and haplotype analyses allowed us to shortlist six candidate genes for four important QTL regions affecting the peduncle vascular bundle traits, including one cloned gene (NAL1) and three newly identified QTL ( $q L V N 6$, qSVN7, and qSVA8.1). Further the most likely candidate genes for each important QTL were also discussed based on functional annotation.

Conclusions: Genetic base on peduncle vascular bundle related traits in rice was systematically dissected, and most likely candidate genes of the known gene NAL1 and the three newly identified QTL (qLVN6, aSVN7, and qSVA8.1) were analyzed. The results provided valuable information for future functional characterization and rice breeding for high yield through optimizing transportation efficiency of photosynthetic products by marker-assisted selection.
\end{abstract}

Keywords: GWAS, Vascular bundle, SNPs, Candidate genes, Quantitative trait loci/locus (QTL)

\section{Background}

Rice (Oryza sativa L.) is one of the most important cereal crops and a staple food for more than one-half of the world's population. In retrospect of rice breeding history, good harmony of source, sink and translocation capacity (i.e. flow) of assimilates plays an important role in improvement of rice yield potential (Donald 1968; Lafitte and Travis 1984; Ashraf et al. 1994). Among them, three top leaves especially flag leaf are main source traits of assimilate synthesis while spikelets

\footnotetext{
*Correspondence: wangyunli55555@163.com; xujianlong@caas.cn ${ }^{1}$ Rice Research Institute, Shenyang Agricultural University/Key Laboratory of Northern Japonica Rice Genetics and Breeding, Ministry of Education, Shenyang 110866, China

${ }^{2}$ Institute of Crop Sciences/National Key Facility for Crop Gene Resources and Genetic Improvement, Chinese Academy of Agricultural Sciences, 12\# South Zhong-Guan-Cun Street, Haidain District, Beijing 100081, China Full list of author information is available at the end of the article
}

including grain number and size are main traits of accumulation of assimilate. The vascular bundle especially in the peduncle is the transport system that links source and sink, and it determines how plants efficiently transport photosynthetic products, mineral nutrients and water from source to sink (Housley and Peterson 1982). Rice breeding history indicated that good balance among source, sink and flow of assimilates, namely, large sink, sufficient source and flow fluency is prerequisite for high yield potential of rice (Ashraf et al. 1994).

The vascular bundle systems in plants play a significant role in delivering water and nutrients as well as other substances needed for growth and defense (Lucas et al. 2013). Vascular bundles, which interconnect all parts of the plant, extending from the stem into leaves, and down into the root system. It consists of xylem and phloem, two differentiated conductive tissues, as well as 
undifferentiated cambial or procambial stem cells. Xylem transports water and dissolved minerals, whereas phloem is required for the distribution of mainly photosynthetic products (sugars, RNA, proteins, and other organic compounds) from source to sink organs (Dettmer et al. 2009). A significant positive correlation between grain yield and the number of vascular bundles and the area of phloem in peduncle have been reported in rice (Ashraf et al. 1994), wheat (Evans et al. 1970) and oat (Housley and Peterson. 1982). The capacity of the vascular bundle system for efficiently transporting assimilates from the source to the sink has been shown as a limiting factor for yield potential realization (Housley and Peterson 1982). Additionally, the differentiation in vascular bundle system is an important parameter that defines differentiation between indica and japonica rice (Fukuyama et al. 1996). Generally, indica varieties tend to have more number of large vascular bundle (LVN) than japonica varieties, though there is no significant difference in the number of small vascular bundle (SVN) between them. The exploitation of the great heterosis that exists in the inter-subspecific crosses between indica and japonica rice has long been considered as a promising way to increase the yield potential. It is therefore of importance to study the genetic basis of peduncle vascular bundle to provide useful information for the genetic improvement of rice yield potential using heterosis between indica and japonica.

The use of QTL mapping has contributed to a better understanding of the genetic basis of many agronomic important traits. For past decades, many researchers have identified QTL for peduncle vascular bundle related traits in rice (Sasahara et al. 1999; Zhang et al. 2002; Cui et al. 2003; Bai et al. 2012). Notably, several QTL affecting the vascular bundle system in rice have been further cloned. For instance, genes such as APO1 (Terao et al. 2010), $A V B$ (Ma et al. 2017) and NAL1 (Qi et al. 2008; Fujita et al. 2013) have been reported to involve in enhanced translocation capacity of vascular bundles.

Cloning QTL affecting complex traits has been a major challenge to plant geneticists and molecular biologists since the classical strategy using map-based cloning of QTL is extremely troublesome and timeconsuming. In recent years, GWAS, based on the genome sequencing and SNP chip technology, makes large-scale high-precision identification to the alleles and the variation which are widely distributed in natural groups of rice become reality (Huang et al. 2010; Zhao et al. 2011; Han and Huang. 2013). In the present study, we reported the first GWAS for vascular bundle system in any agricultural crop using a panel of germplasm resources with a broad genetic diversity. A diverse panel consisting of 423 accessions selected from 3 K Rice Genome Project (3 K RGP) (3K RGP 2014; Sun et al. 2017) was evaluated in 2015 and 2016. GWAS was performed using $27 \mathrm{~K}$ SNPs generated from 3 K RGP through highthroughput sequencing technologies (Zheng et al. 2015). And then the candidate genes based on the SNPs significantly correlated to the vascular bundles through GWAS can be identified.

\section{Results}

Trait variance and correlations

In general, most of the vascular bundle related traits in peduncle appeared to be normally distributed, but a few traits, phloem area of large vascular bundle (LVPA) and phloem area of small vascular bundle (SVPA) showed skewed distributions in 2016 (Fig. 1a). The panel presented substantial variations for all the measured traits. The phenotype pairwise correlations between the measured traits were similar in both years. In 2015 and 2016, strong positive correlations were observed for all area related traits (total area, phloem area, and xylem area) between large and small vascular bundle, indicating the consistent effects of vascular bundle on these traits across all accessions. As expected, the vascular bundle total area had significant positive correlations with its corresponding component traits (phloem area and xylem area) whereas no significant correlations were detected between the vascular bundle total area and the number of vascular bundle (Fig. 1b).

\section{Basic statistics of markers}

For the 26,097 high quality SNPs, the number of markers per chromosome ranged from 1600 on chromosome 9 to 3101 on chromosome 1. The size of chromosome varied from $22.93 \mathrm{Mb}$ for chromosome 9 to 43.25 $\mathrm{Mb}$ for chromosome 1. The whole genome size was $372.50 \mathrm{Mb}$. The average marker spacing was $14.27 \mathrm{~kb}$ with spacing ranging from $12.68 \mathrm{~kb}$ for chromosome 10 to $15.50 \mathrm{~kb}$ for chromosome 4 (Additional file 1: Figure S1).

\section{Population structure}

The screen plot showed that two principal component (PC) can be reserved as informative (Fig. 2a-b; Additional file 2: Table S1), where downhill changed gradually (Fig. 2a). The score plot of PC showed the distribution of each accession in the rice diversity panel (Fig. 2b). Additionally, the highest log likelihood scores of the population structure were observed when the number of populations was set at $2(K=2$; Fig. 2c), indicating that there were two distinct subpopulations (Pop I and Pop II) in the current panel (Fig. 2d; Additional file 2: Table S1). And the heat 


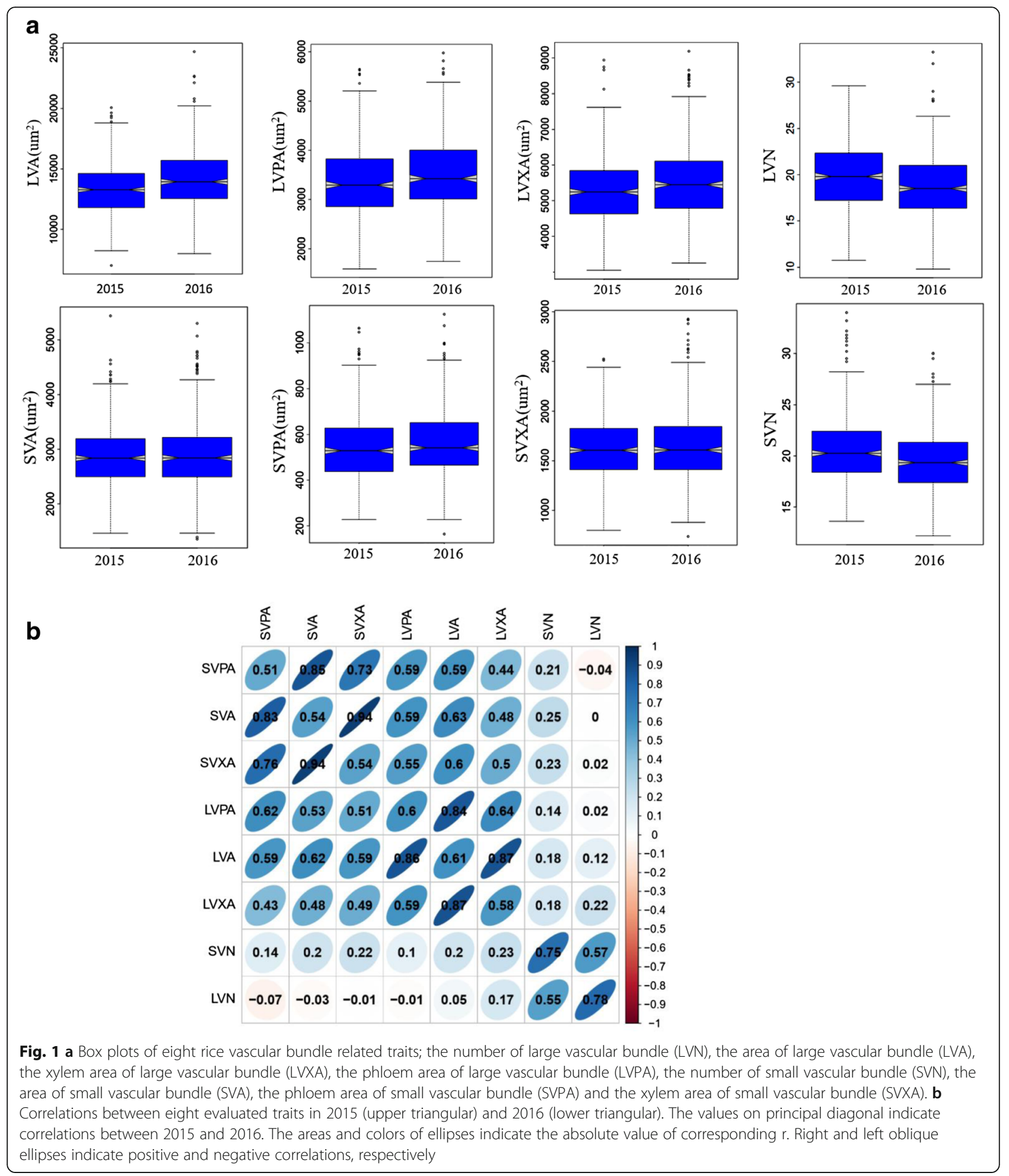



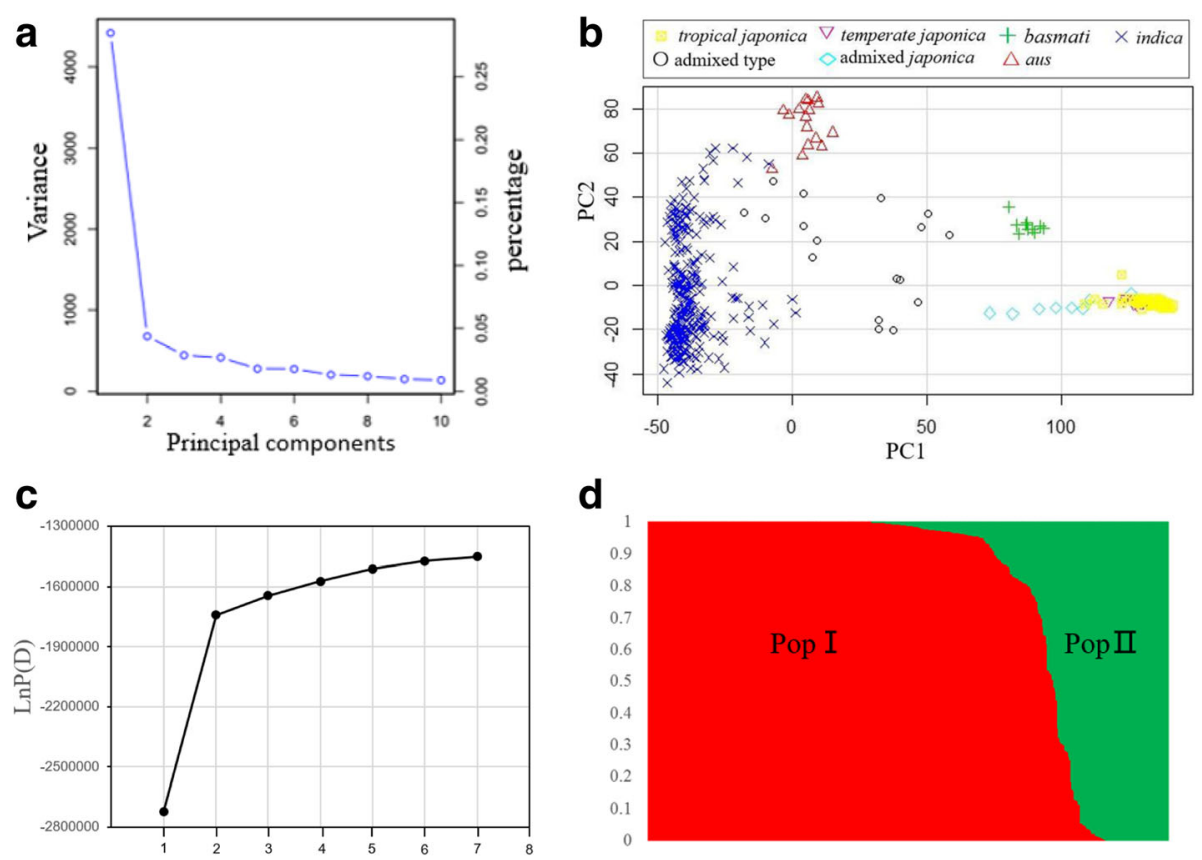

d

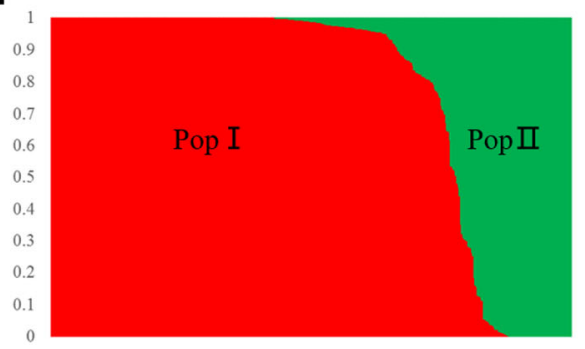

\section{e}

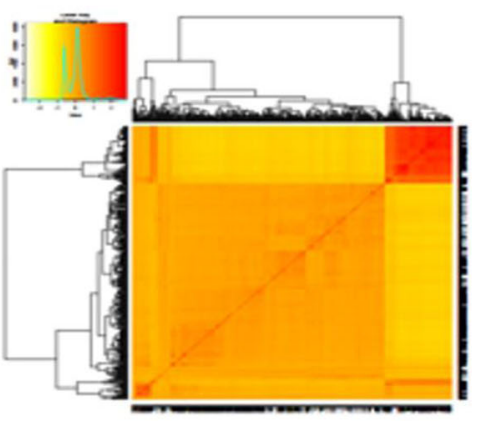

Fig. 2 a Screen plot from GAPIT showing the selection of PCS for association study. b PCA plot based on the screen plot in the rice diversity panel. c Screen plot from STRUCTURE showing the selection of Q. d Bayesian clustering of 423 accessions using STRUCTURE program. e Heat map of kinship from GAPIT with the tree shown on the top and left

map of kinship relatedness matrix (KI) showed the same result (Fig. 2e).

\section{Detection of QTL by GWAS}

A total of 48 different QTL for all traits were identified in 2015 and 2016, ranging from two QTL for SVPA to 10 QTL for LVPA. Among them, 23 (37) QTL were detected in 2015 (2016) and 12 QTL were commonly detected in two years (Table 1; Additional file 3: Figure S2).

For LVN, seven QTL were detected on chromosomes 1, 2, 4, 6 and 11. Among them, qLVN2.1 and qLVN4 were detected in 2015, and accounted for $2.4 \%$ and $2.2 \%$ of phenotypic variance, respectively. Four QTL, $q L V N 1$, $q L V N 2.2, q L V N 2.3$ and $q L V N 11$, were detected in 2016, and explained $2.2 \%$ to $3.0 \%$ of phenotypic variance. Only qLVN6 was detected in both 2015 and 2016, and explained the average phenotypic variance of $2.2 \%$ (Table 1; Additional file 3: Figure S2).

For total area of large vascular bundle (LVA), seven QTL were detected on chromosomes 1, 3, 4, 6, 8 and 11 . The qLVA6 with $3.4 \%$ of phenotypic variance was only detected in 2015 and three QTL (qLVA1, qLVA3, and qLVA4.2) were only detected in 2016, which were accounted for phenotypic variance from $3.5 \%$ to $3.9 \%$. Three QTL, qLVA4.1, qLVA8 and qLVA11, were detected in both years and explained the average phenotypic variance of $2.6 \%, 3.1 \%$ and $3.2 \%$, respectively (Table 1 ; Additional file 3: Figure S2).

Ten QTL for LVPA were detected on chromosomes 1, 2, 4, 8 and 11. Three QTL (qLVPA8.2, qLVPA8.3 and qLVPA11) were detected in 2015, and accounted for $3.2 \%$ to $3.6 \%$ of phenotypic variance. Five QTL (qLVPA1.1, qLVPA1.2, qLVPA2, qLVPA4.2 and qLVPA8.1) 
Table 1 QTL identified for eight peduncle vascular bundle related traits in two years

\begin{tabular}{|c|c|c|c|c|c|c|c|}
\hline Trait $^{\mathrm{a}}$ & QTL & Year & Peak SNP & Allele ${ }^{b}$ & $P$ & Effect $^{c}$ & $R^{2}(\%)^{d}$ \\
\hline \multirow[t]{8}{*}{ LVN } & qLVN1 & 2016 & Chr1-13,801,593 & T/A & $7.8 \mathrm{E}-05$ & -0.82 & 2.4 \\
\hline & qLVN2.1 & 2015 & Chr2-31,993 & $\mathrm{A} / \mathrm{G}$ & $4.8 \mathrm{E}-05$ & -0.89 & 2.4 \\
\hline & qLVN2.2 & 2016 & Chr2-25,871,069 & $A / G$ & $5.5 \mathrm{E}-05$ & 1.64 & 2.5 \\
\hline & qLVN2.3 & 2016 & Chr2-29,521,254 & $\mathrm{T} / \mathrm{C}$ & $8.8 \mathrm{E}-05$ & -1.56 & 2.2 \\
\hline & qLVN4 & 2015 & Chr4-5,193,957 & $\mathrm{G} / \mathrm{A}$ & $8.1 \mathrm{E}-05$ & 0.77 & 2.2 \\
\hline & qLVN6 & 2015 & Chr6-28,662,981 & $C / T$ & $9.4 \mathrm{E}-05$ & -0.67 & 2.0 \\
\hline & & 2016 & Chr6-28,639,502 & $\mathrm{G} / \mathrm{C}$ & $9.5 \mathrm{E}-05$ & -0.51 & 2.4 \\
\hline & qLVN11 & 2016 & Chr11-1,063,214 & $C / T$ & $1.3 \mathrm{E}-05$ & 1.37 & 3.0 \\
\hline \multirow[t]{10}{*}{ LVA } & qLVA1 & 2016 & Chr1-2,179,066 & $A / G$ & 4.4E-05 & -617.1 & 3.8 \\
\hline & qLVA3 & 2016 & Chr3-33,078,718 & T/C & $8.2 \mathrm{E}-05$ & -714.3 & 3.5 \\
\hline & qLVA4.1 & 2015 & Chr4-31,081,174 & $C / A$ & 9.6E-05 & -392.1 & 1.4 \\
\hline & & 2016 & Chr4-31,068,550 & $\mathrm{G} / \mathrm{C}$ & 4.1E-05 & -1073.3 & 3.8 \\
\hline & qLVA4.2 & 2016 & Chr4-32,189,616 & T/C & $3.8 \mathrm{E}-05$ & -960.8 & 3.9 \\
\hline & qLVA6 & 2015 & Chr6-20,057,366 & $C / T$ & $6.1 \mathrm{E}-05$ & 1362.6 & 3.4 \\
\hline & qLVAB & 2015 & Chr8-3770 & $\mathrm{G} / \mathrm{A}$ & $6.4 \mathrm{E}-05$ & -524.4 & 2.5 \\
\hline & & 2016 & Chr8-69,600 & $C / T$ & $6.4 \mathrm{E}-05$ & -620.0 & 3.6 \\
\hline & qLVA11 & 2015 & Chr11-21,798,323 & T/G & 7.1E-05 & 463.6 & 3.0 \\
\hline & & 2016 & Chr11-21,798,323 & $T / G$ & $9.0 \mathrm{E}-05$ & 582.6 & 3.4 \\
\hline \multirow[t]{12}{*}{ LVPA } & qLVPA1.1 & 2016 & Chr1-1,806,093 & $C / A$ & $6.4 \mathrm{E}-05$ & 252.9 & 3.5 \\
\hline & qLVPA1.2 & 2016 & Chr1-2,179,066 & $A / G$ & 8.7E-05 & -175.1 & 3.4 \\
\hline & qLVPA2 & 2016 & Chr2-30,012,958 & $C / G$ & $8.0 \mathrm{E}-05$ & -508.7 & 3.4 \\
\hline & qLVPA4.1 & 2015 & Chr4-31,081,174 & $C / A$ & $6.7 \mathrm{E}-05$ & -83.1 & 3.0 \\
\hline & & 2016 & Chr4-31,068,550 & $\mathrm{G} / \mathrm{C}$ & $3.0 \mathrm{E}-05$ & -322.3 & 3.9 \\
\hline & qLVPA4.2 & 2016 & Chr4-32,189,616 & T/C & $6.1 \mathrm{E}-05$ & -277.5 & 3.6 \\
\hline & qLVPA8.1 & 2016 & Chr8-3770 & $\mathrm{G} / \mathrm{A}$ & $4.5 \mathrm{E}-05$ & -219.5 & 3.7 \\
\hline & qLVPA8.2 & 2015 & Chr8-2,393,328 & T/A & $3.8 \mathrm{E}-05$ & -524.5 & 3.6 \\
\hline & qLVPA8.3 & 2015 & Chr8-27,101,483 & $C / T$ & $9.5 \mathrm{E}-05$ & 220.4 & 3.2 \\
\hline & qLVPA8.4 & 2015 & Chr8-27,737,011 & $C / T$ & $2.9 \mathrm{E}-05$ & 187.6 & 3.7 \\
\hline & & 2016 & Chr8-27,707,779 & $C / T$ & $9.2 \mathrm{E}-05$ & -190.4 & 2.8 \\
\hline & qLVPA11 & 2015 & Chr11-25,611,048 & $C / A$ & $3.9 \mathrm{E}-05$ & -360.2 & 3.5 \\
\hline \multirow[t]{11}{*}{ LVXA } & qLVXA1 & 2016 & Chr1-2,758,104 & $A / G$ & $5.8 \mathrm{E}-05$ & -514.4 & 3.7 \\
\hline & qLVXA5 & 2015 & Chr5-4,823,527 & $C / T$ & $8.4 \mathrm{E}-05$ & -224.9 & 3.2 \\
\hline & & 2016 & Chr5-4,823,527 & $C / T$ & $3.4 \mathrm{E}-05$ & -287.8 & 3.9 \\
\hline & $q L V X A G$ & 2016 & Chr6-21,044,730 & $C / A$ & $5.9 \mathrm{E}-05$ & 415.6 & 3.7 \\
\hline & qLVXA8.1 & 2015 & Chr8-87,741 & T/C & $5.4 \mathrm{E}-05$ & 220.6 & 2.8 \\
\hline & & 2016 & Chr8-69,600 & $C / T$ & $1.4 \mathrm{E}-06$ & -325.9 & 5.3 \\
\hline & qLVXA8.2 & 2015 & Chr8-220,938 & $C / T$ & $3.0 \mathrm{E}-05$ & 195.8 & 2.3 \\
\hline & & 2016 & Chr8-220,938 & $C / T$ & 2.7E-05 & 282.4 & 4.0 \\
\hline & qLVXA8.3 & 2015 & Chr8-27,031,242 & T/A & $8.6 \mathrm{E}-05$ & 439.9 & 3.2 \\
\hline & qLVXA11 & 2015 & Chr11-21,798,323 & T/G & $1.6 \mathrm{E}-05$ & 245.6 & 3.9 \\
\hline & & 2016 & Chr11-21,798,323 & T/G & 2.7E-05 & 222.9 & 2.7 \\
\hline \multirow[t]{3}{*}{ SVN } & qSVN1 & 2016 & Chr1-9,724,654 & T/C & $4.3 \mathrm{E}-05$ & -1.76 & 3.6 \\
\hline & qSVN2 & 2016 & Chr2-25,915,457 & $C / A$ & $5.5 \mathrm{E}-05$ & -1.98 & 3.5 \\
\hline & qSVN5.1 & 2015 & Chr5-24,874,414 & $\mathrm{A} / \mathrm{G}$ & $3.3 \mathrm{E}-05$ & -1.69 & 3.9 \\
\hline
\end{tabular}


Table 1 QTL identified for eight peduncle vascular bundle related traits in two years (Continued)

\begin{tabular}{|c|c|c|c|c|c|c|c|}
\hline Trait $^{a}$ & QTL & Year & Peak SNP & Allele $^{b}$ & $P$ & Effect $^{c}$ & $R^{2}(\%)^{d}$ \\
\hline & & 2016 & Chr5-24,874,414 & $A / G$ & $2.7 \mathrm{E}-06$ & -1.77 & 4.7 \\
\hline & qSVN5.2 & 2016 & Chr5-25,915,442 & $\mathrm{G} / \mathrm{A}$ & 5.7E-05 & 1.97 & 3.5 \\
\hline & qSVN7 & 2015 & Chr7-29,604,076 & $C / T$ & 4.7E-05 & 1.27 & 2.8 \\
\hline & & 2016 & Chr7-29,604,076 & $C / T$ & $1.9 \mathrm{E}-06$ & 1.69 & 4.9 \\
\hline \multirow[t]{6}{*}{ SVA } & QSVA2.1 & 2015 & Chr2-10,294,412 & $\mathrm{G} / \mathrm{A}$ & $8.0 \mathrm{E}-05$ & 139.9 & 3.7 \\
\hline & qSVA2.2 & 2016 & Chr2-11,856,232 & $C / A$ & 3.7E-05 & -342.0 & 3.7 \\
\hline & qSVA8.1 & 2016 & Chr8-2,031,279 & $C / T$ & $3.0 \mathrm{E}-05$ & -258.4 & 3.8 \\
\hline & qSVA8.2 & 2016 & Chr8-20,982,368 & $\mathrm{G} / \mathrm{T}$ & $7.9 \mathrm{E}-05$ & -251.3 & 3.4 \\
\hline & qSVA11 & 2015 & Chr11-25,611,048 & $C / A$ & 6.67E-05 & -303.8 & 3.6 \\
\hline & qSVA12 & 2016 & Chr12-3,630,490 & $C / T$ & 5.17E-05 & -234.6 & 3.5 \\
\hline \multirow[t]{2}{*}{ SVPA } & 9SVPA4.1 & 2016 & Chr4-13,422,276 & $C / T$ & $6.53 \mathrm{E}-05$ & 110.0 & 3.7 \\
\hline & qSVPA4.2 & 2016 & Chr4-31,878,154 & $A / G$ & $3.56 \mathrm{E}-05$ & 38.7 & 3.9 \\
\hline \multirow[t]{4}{*}{ SVXA } & QSVXA2 & 2016 & Chr2-30,012,958 & $C / G$ & $6.4 \mathrm{E}-05$ & -265.8 & 3.5 \\
\hline & QSVXAB & 2016 & Chr8-2,031,279 & $C / T$ & $9.74 \mathrm{E}-05$ & -145.4 & 3.4 \\
\hline & QSVXA9 & 2015 & Chr9-16,464,178 & $C / T$ & $1.28 \mathrm{E}-05$ & -149.5 & 4.4 \\
\hline & qSVXA11 & 2015 & Chr11-25,611,048 & $C / A$ & 1.13E-05 & -88.2 & 4.5 \\
\hline
\end{tabular}

a LVN the number of large vascular bundle, LVA the total area of large vascular bundle; LVPA the phloem area of large vascular bundle, LVXA the xylem area of large vascular bundle, SVN the number of small vascular bundle, SVA the total area of small vascular bundle, SVPA the phloem area of small vascular bundle,

SVXA the xylem area of small vascular bundle

${ }^{\mathrm{b}}$ Major/Minor allele

${ }^{\mathrm{C}}$ Effect: Allele effect with respect to the minor allele

${ }^{d} R^{2}(\%)$ : Phenotypic variance explained

were detected in 2016 and accounted for phenotypic variance from $3.4 \%$ to $3.7 \%$. Another two QTL, qLVPA4.1 and $q L V P A 8.4$ were commonly detected in the two years, and explained the average phenotypic variance $3.5 \%$ and $3.3 \%$, respectively (Table 1 ; Additional file 3: Figure S2).

For xylem area of large vascular bundle (LVXA), seven QTL were detected on chromosomes 1, 5, 6, 8, and 11 . Among them, only qLVXA8.3 was detected in 2015 and explained 3.2\% of phenotypic variance. Two QTL, qLVXA1 and qLVXA6, were detected in 2016 and accounted for both $3.7 \%$ of phenotypic variance. Four QTL, namely, qLVXA5, qLVXA8.1, qLVXA8.2 and qLVXA11, were simultaneously detected in the two years and explained the average phenotypic variance of $3.6 \%$, $4.1 \%, 3.2 \%$ and $3.3 \%$, respectively (Table 1; Additional file 3: Figure S2).

For SVN, five QTL were detected on chromosomes 1, 2,5 , and 7 , including three (qSVN1, qSVN2 and qSVN5.2) detected in 2016 and two (qSVN5.1 and qSVN7) in the two years. The first three QTL accounted for phenotypic variance from $3.5 \%$ to $3.6 \%$ while the latter two QTL explained the average phenotypic variance of $4.3 \%$ and $3.9 \%$, respectively (Table 1; Additional file 3 : Figure S2).

For total area of small vascular bundle (SVA), a total of six QTL were detected on chromosomes 2, 8, 11, and 12. Two QTL (qSVA2.1 and qSVA11) were detected in
2015, and accounted for $3.7 \%$ and $3.6 \%$ of phenotypic variance, respectively. The other four QTL (qSVA2.2, qSVA8.1, qSVA8.2 and qSVA12) were detected in 2016, and accounted for $3.4 \%$ to $3.8 \%$ of phenotypic variance (Table 1; Additional file 3: Figure S2).

For SVPA, two QTL, qSVPA4.1 and qSVPA4.2 were detected on chromosome 4 in 2016, and accounted for $3.7 \%$ and $3.9 \%$ of phenotypic variance, respectively (Table 1; Additional file 3: Figure S2).

For xylem area of small vascular bundle (SVXA), four QTL were detected on chromosomes 2, 8, 9 and 11. Of them, two QTL ( $q S V X A 9$ and qSVXA11) were detected in 2015 and accounted for $4.4 \%$ and $4.5 \%$ of phenotypic variance, respectively. Another two QTL (qSVXA2 and qSVXA8) were detected in 2016 and explained 3.5\% and $3.4 \%$ of phenotypic variance, respectively (Table 1; Additional file 3: Figure S2). There was no any QTL simultaneously detected in the two years.

\section{Candidate gene analysis}

For eight peduncle vascular bundle related traits, we conducted gene-based association and haplotype analysis to identify candidate genes for important QTL identified using the whole population. In addition, considering that among the eight peduncle vascular bundle related traits LVN, SVA, SVXA and SVPA were both significantly different between indica and japonica in 2015 and 2016 (Additional file 4: Figure S3), we conducted haplotype 

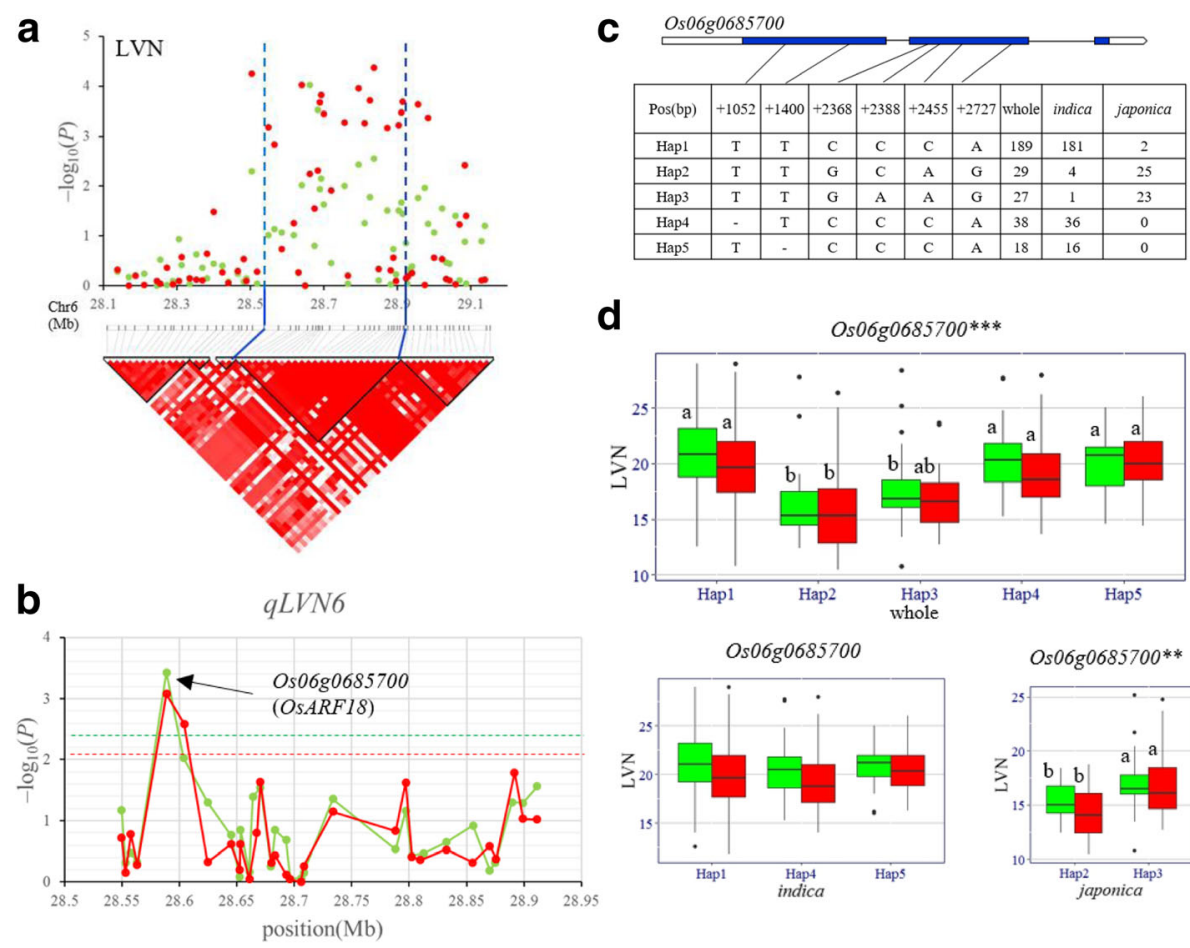

d
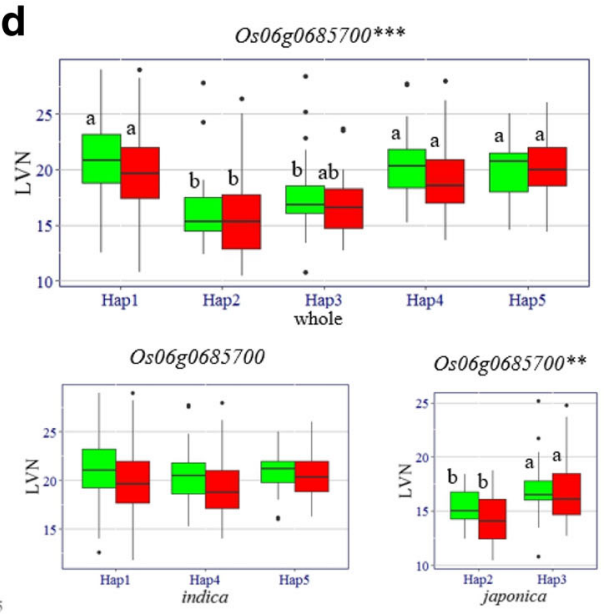

Fig. 3 Gene-based association analysis and haplotype analysis of targeted genes related to qLVN6. a Local Manhattan plot (top) and LD block (bottom) surrounding the peak on chromosome 6. Dashed lines indicate the candidate region for the peak. $\mathbf{b}$ Gene-based association analysis of targeted genes related to qLVN6. Each point is a gene indicated by one SNP having largest-log10 $(P)$ value. Dash line show the threshold to determine significant SNP. c Exon-intron structure of Os06g0685700 and DNA polymorphism in that gene. $\mathbf{d}$ Boxplots for LVN based on the haplotypes (Hap) for Os06g0685700 in the whole, indica and japonica populations. The ** and ${ }^{* * *}$ suggest significance of ANOVA at $P<0.01$ and $P<0.001$, respectively. The letter on histogram ( $a$, and $b$ ) indicate multiple comparisons result at the significant level 0.05 . Green and red colors indicate in 2015 and 2016, respectively

analysis of targeted genes related to LVN, SVA, SVXA and SVPA in indica and japonica subpopulations, respectively.

Using gene-based association analysis and haplotype analysis of candidate gene, six important candidate genes were identified for the four QTL regions distributing on chromosomes 4, 6, 7 and 8, ranging from one to two candidate genes for each QTL. For $q L V N 6$ in the candidate region of $28.55-28.92 \mathrm{Mb}(370 \mathrm{~kb})$ on chromosome 6 (Fig. 3a), 616 non-synonymous SNPs of 52 genes were used for association analysis according to the Rice Genome Annotation Project Database (RAP-DB). The peak SNP was at 28588881 bp on chromosome 6 with $-\log _{10}(P)$ of 3.43 and 3.08 in 2015 and 2016, respectively. Again, SNPs with $-\log _{10}(P)$ above the threshold (2.43 in 2015 and 2.08 in 2016) were found to locate in one gene, Os06g0685700 (Fig. 3b). Significant differences for LVN between different haplotypes were observed at $0 s 06 g 0685700$ in whole population (Fig. 3c-d).In this study, haplotype was separately analyzed for indica and japonica varieties owing to the significant differentiation in LVN between the two subspecies.
Highly significant differences in LVN were detected between different haplotypes at the candidate gene (Os06g0685700) in japonica, though there was no significant difference for LVN between different haplotypes in indica accessions (Fig. 3c-d).

Similarly, the candidate region was predicted in the region from 31.07 to $31.26 \mathrm{Mb}(190 \mathrm{~kb})$ on chromosome 4 harboring $q L V A 4.1$ and $q L V P A 4.1$ (Fig. 4a), and 303 non-synonymous SNPs of 30 genes were used for association analysis. The peak SNP was at 31079854 bp with $-\log _{10}(P)$ of 3.73 (3.47) in 2015 (2016) for LVA, and 3.49 (3.21) in 2015 (2016) for LVPA. Two genes harboring SNPs with $-\log _{10}(P)$ larger than the thresholds were identified, including Os04g0613000 and Os04g0615000 (Fig. 4b). Highly significant differences for qLVA4.1 and qLVPA4.1 between different haplotypes were observed at the two genes (Os04g0613000 and Os04g0615000) in whole population (Fig. 4c-d). Of the two genes, Os04g0615000 is identical to NAL1 (NARROW $L E A F 1)$, the gene regulating the development of vascular bundle in previously reported (Qi et al. 2008). Three haplotypes of NAL1 were found and haplotype 


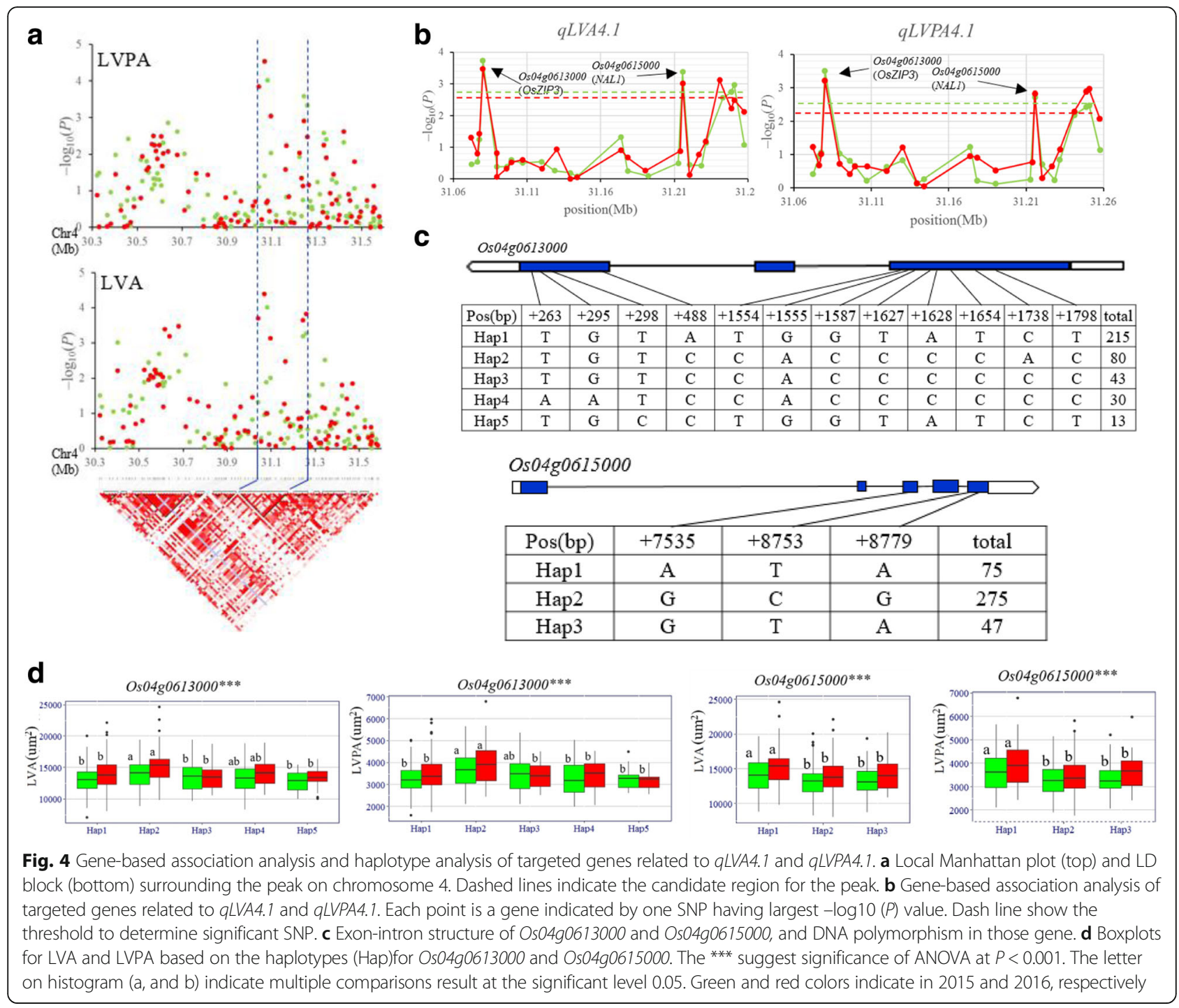

ATA was associated with significantly larger LVA and LVPA than haplotypes GCG and GTA (Fig. 4c-d).

QSVN7 was fine-mapped in the region of 29.46$29.67 \mathrm{Mb}(210 \mathrm{~kb})$ on chromosome 7 (Fig. 5a), and 353 non-synonymous SNPs of 28 genes were used for association analysis. The peak SNP for $q S V N 7$ was at 29626817 bp on chromosome 7 with $-\log _{10}(P)$ of 3.57 and 3.34 in 2015 and 2016, respectively. SNPs with $-\log _{10}$ $(P)$ above the thresholds were located in the two candidate genes, Os07g0695100 and Os07g0694700 (Fig. 5b). No significant haplotypes were found in Os07g0694700. Five haplotypes were found for Os07g0695100. Haplotypes analysis revealed that significant differences for SVN were observed between haplotypes GCGGTAGCGG and CTGACGGTGA in both years (Fig. $5 \mathrm{c}-\mathrm{d}$ ).

On chromosome 8, a high peak of qSVA8.1 was found to be overlapped with the peak of qSVXA8. We predicted the candidate region from 1.99-2.17 Mb (180 kb)
(Fig. 6a), and 277 non-synonymous SNPs of 29 genes were used for association analysis. The peak SNP was at $2079621 \mathrm{bp}$ on chromosome 8 with $-\log _{10}(P)$ of 3.56 for $q S V A 8.1$ and 3.50 for $q S V X A 8$ in 2016. Two genes harboring the SNPs with $-\log _{10}(P)$ larger than the threshold (2.56 and 2.50 for qSVA8.1 and qSVXA8, respectively) were identified, including Os08g0137100 and Os08g0137250 (Fig. 6b). Significant differences were detected among different haplotypes at the two genes in whole population (Fig. 6c-d). However, no significant difference in SVA and SVXA was detected between different haplotypes at the two candidate genes in indica subpopulation, and only one prevalent haplotype of each gene was detected in japonica subpopulation (Additional file 5: Table S2). Among them, OsO8g0137100 is identical to OsFIE2, a gene affecting vascular bundle related traits in previously report (Liu et al. 2016). 

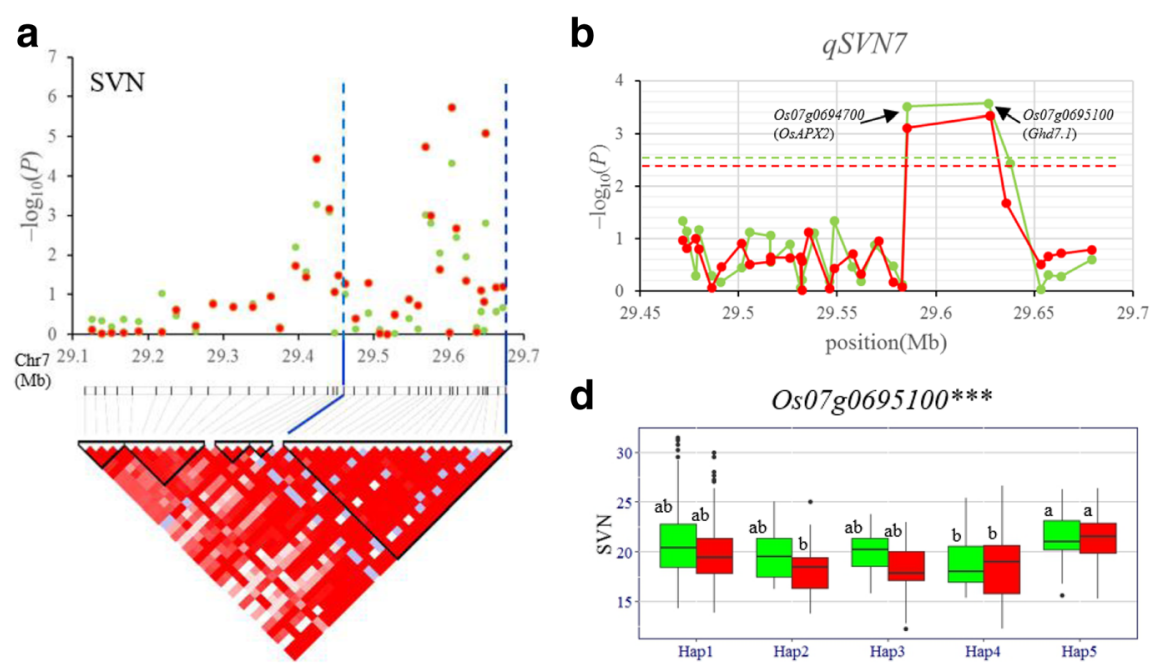

C

Os $07 g 0695100$
\begin{tabular}{|c|c|c|c|c|c|c|c|c|c|c|c|c|}
\hline Pos(bp) & +770 & +848 & +927 & +7071 & +10111 & +10203 & +10315 & +10522 & +10534 & +11104 & total \\
\hline Hap1 & G & C & G & G & C & A & A & C & T & G & 243 \\
\hline Hap2 & G & C & G & G & C & A & G & C & G & G & 22 \\
\hline Hap3 & G & T & T & A & C & G & G & T & G & G & 19 \\
\hline Hap4 & G & C & G & G & T & A & G & C & G & G & 26 \\
\hline Hap5 & C & T & G & A & C & G & G & T & G & A & 22 \\
\hline
\end{tabular}

Fig. 5 Gene-based association analysis and haplotype analysis of targeted genes related to qSVN7. a Local Manhattan plot (top) and LD block (bottom) surrounding the peak on chromosome 7. Dashed lines indicate the candidate region for the peak. $\mathbf{b}$ Gene-based association analysis of targeted genes related to aSVN7. Each point is a gene indicated by one SNP having largest -log10 (P) value. Dash line show the threshold to determine significant SNP. c Exon-intron structure of Os07g0695100 and DNA polymorphism in that gene. $\mathbf{d}$ Boxplots for LVN based on the haplotypes (Hap) for Os07g0695100. The *** suggest significance of ANOVA at $P<0.001$. The letter on histogram $(\mathbf{a}$, and $\mathbf{b})$ indicate multiple comparisons result at the significant level 0.05 . Green and red colors indicate in 2015 and 2016, respectively

\section{Discussion}

\section{Characteristics of vascular bundle between Indica and Japonica}

Indica and japonica are the two major types of Asian cultivated rice (Oryza sativa L.). There are significant differences between them in many morphological and physiological traits associated with the processes of indica-japonica differentiation (Morishima and Oka 1981). Among them, the differentiation in vascular bundle system is an important parameter that defines differentiation between the two subspecies. Indica varieties tend to have more large vascular bundles in peduncle than japonica varieties, and have significant higher ratio of the LVN to the number of primary rachis branches (ranged from 1.3 to 2.2) than japonica (nearly1.0) (Fukuyama and Takayama 1995). Our studies showed that LVN in peduncle of indica (averaged 21 in 2015 and 19.9 in 2016) was also significantly higher than those of japonica (averaged 16.1 in 2015 and 15.5 in 2016) (Additional file 4: Figure S3). In japonica-type cultivars, each of the large vascular bundles is directly connected to a primary rachis branch (PRB), resulting almost in same quantity of LVN in the peduncle and number of
PRBs. On the other hand, in indica-type some large vascular bundles are directly connected to the secondary rachis branches due to surplus large vascular bundles (Fukushima and Akita 1997; Terao et al. 2010). The different vascular bundle characteristics between indica and japonica probably explained inherently shorter grain development phase (Osada et al. 1983) or shorter grain filling period (Nagato and Chaudhry 1969) in indica than japonica resulting from more rapid multiplication of endosperm cells (Nagato and Chaudhry 1969) and dominant transportation efficiency of photosynthetic products (Weng and Chen 1987) in indica. Rice grain yield is highly positively significantly correlated with spikelet number on first rachis branches especially secondary rachis branches. So, genetic mechanisms and their genetic relationships between the number of rachis branches and vascular bundle related traits in rice need further explored.

\section{Candidate gene identification of the important QTL}

Cloning QTL affecting complex traits has been a major challenge to plant geneticists and molecular biologists since the classical strategy using map-based cloning is 

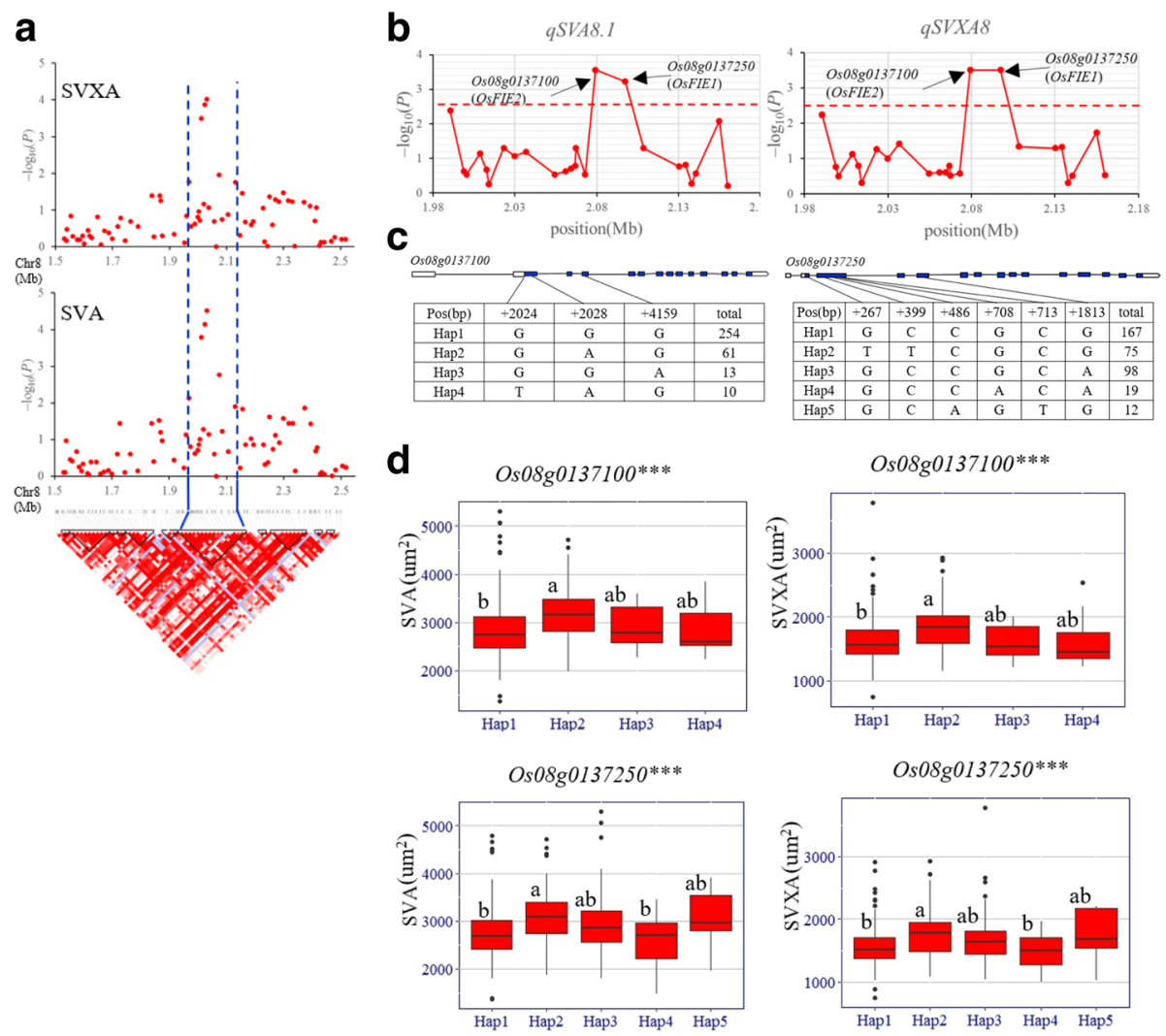

Fig. 6 Gene-based association analysis and haplotype analysis of targeted genes related to qSVA8.1 and qSVXA8. a Local Manhattan plot (top) and LD block (bottom) surrounding the peak on chromosome 8. Dashed lines indicate the candidate region for the peak. b Gene-based association analysis of targeted genes related to qSVA8.1 and GSVXA8. Each point is a gene indicated by one SNP having largest -log $10(P)$ value. Dash line show the threshold to determine significant SNP. c Exon-intron structure of Os08g0137250 and Os08g0137100, and DNA polymorphism in those gene. d Boxplots for SVA and SVXA based on the haplotypes (Hap) for Os08g0137250 and Os08g0137100. The *** suggest significance of ANOVA at $P<0.001$. The letter on histogram $(a$, and $b)$ indicate multiple comparisons result at the significant level 0.05 . Green and red colors indicate in 2015 and 2016, respectively

extremely troublesome and time-consuming. Using GWAS and haplotype analysis of candidate genes, we were able to shortlist six candidate genes governing the four important QTL affecting vascular bundles related traits in the peduncle.

In the region of 31.07-31.26 $\mathrm{Mb}$ on chromosome 4 , a QTL cluster ( $q L V A 4.1$ and $q L V P A 4.1)$ was detected, containing two candidate genes. One of them was NAL1 (Os04g0615000) regulating the development of vascular bundle which may be involved in polar auxin transport (Qi et al. 2008) and contributed to grain yield (Fujita et al. 2013; Xu et al. 2015). Culms of nal1 showed a defective vascular system, in which the number and distribution pattern of vascular bundles was altered $(\mathrm{Qi}$ et al. 2008). Additionally, the Takanari allele of NAL1, which was found to be a partial loss-of-function allele of NAL1, increased mesophyll cell number between vascular bundles (Takai et al. 2013). Haplotypes analysis revealed haplotype ATA was associated with significantly larger LVA and LVPA than haplotypes GCG and GTA
(Fig. 4c-d). Further, we found one nucleotide variation located in the third exon which results in the substitution of a histidine (CAT) in the trypsin-like serine and cysteine protease domain of the large LVA and LVPA by an arginine (CGT) in the small LVA and LVPA (Additional file 6: Fig. S4). And an allele variation produced less panicles per plant, more spikelet numbers and more width leaf blades than varieties containing $G$ allele (Yano et al. 2016). Taguchi-Shiobara et al. (2015) reported that the flag leaf width of transgenic plants of NAL1 varied depending on the ratio of $\mathrm{A}$ allele to $\mathrm{G}$ allele. Thus, we presumed that the nucleotide variation $(\mathrm{A} / \mathrm{G})$ in the third exon exerted a critical function on determining panicles per plant, spikelet numbers per panicle, flag leaf width and area of large vascular bundle in the peduncle.

In the present study, two QTL (qLVN6 and qSVN7) were detected in both two years. The qLVN6 affecting $\mathrm{LVN}$ was identified in the region of $28.55-28.92 \mathrm{Mb}$ on chromosome 6.The only candidate gene for $q L V N 6$ was OsARF18 (Os06g0685700), a similar to auxin response 
factor gene. The deregulation of the OsmiR160 target gene OsARF18 leads to rice abnormal growth and development, including dwarf stature, rolled leaves, and small seeds through affecting the auxin signaling (Huang et al. 2016). Compared with wild type, numbers of bulliform cell and total abaxial epidermal cells between two vascular bundles were significantly reduced in $m O s A R F 18$ leave (Huang et al. 2016). A number of studies have shown that polar auxin transport is required for continuous vascular pattern formation and establishment of procambial strands (Mattsson et al. 2003; Scarpella et al. 2006; Berleth et al. 2007). The classic auxin signal flow canalization hypothesis predicts that the distribution of auxin is narrowed down from a wide field of cells to a subset of cells with high auxin transport, which then become the site of the procambium (Sachs 1981). Then gene function in PINT, MP and ATHB8, which is a positive feedback loop of auxin-MP-ATHB8-PIN1, confirms the hypothesis (Paciorek et al. 2005; Scarpella et al. 2006; Donner et al. 2009). In addition, $A V B$ gene affecting vascular bundles involved in procambium establishment following auxin signaling in lateral primordial $\mathrm{Ma}$ et al. 2017). Previously, researches considered that NAL1 (Os04g0615000) regulating the development of vascular bundle played its role in leaf morphogenesis by regulating polar auxin transport, and plays a regulatory role in the development of plant type in rice (Qi et al. 2008; Fujita et al. 2013). Therefore, OsARF18 (Os06g0685700) is considered as a possible candidate gene of $q L V N 6$. Transgenic experiments are under way to verify the functionalities of the candidate genes. The only candidate gene for qSVN7, Os07g0695100 (Ghd7.1) was a major QTL with pleiotropic effects on grain number per panicle, plant height, and heading date in rice (Oryza sativa L.) in previously report (Liu et al. 2013; Gao et al. 2014). In this study, compared with haplotype GCGGTAGCGG, haplotype CTGACGGTGA delayed heading and increased SVN, grain number per panicle and plant height in 2016 Sanya (Additional file 7: Figure S5), hinting that Ghd7.1 probably affects vascular bundle in rice. However and so far there has been no reported Ghd7.1 gene relevant to vascular bundle. Thus, as to whether Ghd7.1 is candidate gene for $q S V N 7$, it must be verified using reverse genetic approaches.

Of the two candidate genes for qSVA8.1, OsFIE2 (Os08g0137100) belongs to the PcG protein family and is the most likely candidate gene. Compared to wildtype, the number of large and small vascular bundles decreased in second internode of osfie2-1 stem, as well as cell number and cell size in spikelet hulls (Liu et al. 2016). However, no significant difference was detected between different haplotypes at Os08g0137100 in indica subpopulation, and only one haplotype was observed in japonica subpopulation (Additional file 5: Table S2), though there were significant differences for SVA and SVXA between different haplotypes in whole population (Fig. 6d), suggesting that indica-japonica differentiation probably led to significant phenotypic differences in SVA and SVXA between different haplotypes. Thus, it must be further studies that OsFIE2 is candidate gene for qSVA8.1 or evolution gene of indica and japonica.

\section{Application in Rice breeding for improved vascular bundle system}

The grain yield of crop is highly dependent on the source-sink relationship in which grain or kernel is the photosynthetic sink while the leaves are the primary source. The vascular bundle is the transport system that links source and sink, and it determines how plants efficiently transport photosynthetic products, mineral nutrients and water from source to sink (Housley and Peterson 1982). Efficient transport of assimilates from leaves and stems to developing spikelets is required for better grain filling and high yield. SPIKE gene increases grain yield of indica rice through enlarging sink size, source size, and translocation capacity. Compared with that of IR64 isogenic control, the grain fertility of NILSPIKE was improved, presumably owing to a strengthening of assimilate supply to the larger number of spikelets by an increase in vascular bundle number of panicle neck (Fujita et al. 2013). APO1 gene controlling the number of primary rachis branches also controls the vascular bundle formation and hence is responsible to increase the harvest index and grain yield in rice (Terao et al. 2010). The effect on vascular bundle system development regulated by the HI1 allele of APO1 limited reduction in ripening percentage (Terao et al. 2010). In a word, the capacity of transporting assimilates from source to sink could limit grain filling (Ashraf et al. 1994). What rice breeders concern is how to develop new variety with an advanced vascular bundle system and a strong ability to transport assimilates from source to sink.

Our results suggested that in the region of 28.55$28.92 \mathrm{Mb}$ on chromosome 6, a QTL (qLVN6) was detected in both two years affecting LVN. According to gene function annotation, Os06g0685700, a similar to auxin response factor gene is considered as the most likely candidate gene of $q L V N 6$. Haplotype analysis revealed that significant differences for LVN were between different haplotypes at the gene in japonica accessions. Two tropical japonica varieties, PLUS (averaged 20.4 in 2015 and 23.5 in 2016) and ITA 221 (averaged 21.7 in 2015 and 23.7 in 2016) with more LVNs were found in this panel. Haplotype TTGAAG of the Os06g0685700 from the two japonica accessions exhibited additive effect for increased number of large vascular bundles in peduncle in japonica and this favorable QTL allele 
would be introgressed into indica and other japonica varieties with superior agronomic traits for improved large vascular bundle by marker-assisted selection (MAS). Specifically, for example, hybrid between indica and japonica holds large panicle with more primary rachis branches especially secondary rachis branches but its grain yield is seriously limited by insufficient grain filling due to disharmony of source, sink and flow of assimilates. So, it is possible to improve grain filling of hybrid between two subspecies by reasonably deploying favorable alleles of QTL for peduncle vascular bundle related traits in rice as indicated in this study.

\section{Conclusion}

We identified 48 QTL for eight peduncle vascular bundle related traits via GWAS. A total of six candidate genes of four important QTL regions, including the cloned gene (NAL1), were identified by determining linkage disequilibrium blocks associated with significant SNPs, and haplotype analyses. In addition, the most likely candidate genes for three new QTL ( $q L V N 6$, qSVN7, and qSVA8.1) were identified based on functional annotation. The results will enhance our knowledge of the genetic basis of vascular bundle traits in rice, facilitate exploring the molecular mechanisms underlying the traits, and provide valuable information for improving rice vascular bundle system by MAS breeding.

\section{Methods}

\section{Plant materials}

The 423 worldwide accessions were selected from $3 \mathrm{~K}$ RGP (Zheng et al. 2015). This panel consisted of seven types, including indica (301), temperate japonica (13), tropical japonica (57), admixed japonica (9), admixed type (17), aus/boro (16), and basmati/sadri (10) (Additional file 2: Table S1).

\section{Phenotypic investigation}

All of these accessions were grown in Sanya $\left(18.3^{\circ} \mathrm{N}\right.$, 109.3 E) during Dec 2014-April 2015 and Dec 2015April 2016 and each accession was planted in two rows with 10 individuals each row at a spacing of $25 \mathrm{~cm}$ between rows and $17 \mathrm{~cm}$ between plants with two replications for each accession. The field management followed the farmers' standard management practices. In order to minimize flowering time effects experiment-wise, batch sampling were performed based on heading date of each accession. At full heading stage, five uniform plants in the middle of each plot were selected. The transverse section of stem were made at uniformly $2 \mathrm{~cm}$ above the neck-panicle node and kept in formalin-acetic-alcohol (FAA) fixative solution. After safranin O staining, vascular bundle related traits in the peduncle, including the number of large vascular bundle (LVN), the total area of large vascular bundle (LVA, $\mathrm{um}^{2}$ ), the phloem area of large vascular bundle (LVPA, $\mathrm{um}^{2}$ ), the xylem area of large vascular bundle (LVXA, um ${ }^{2}$ ), the number of small vascular bundle $(\mathrm{SVN})$, the total area of small vascular bundle (SVA, um ${ }^{2}$ ), the phloem area of small vascular bundle (SVPA, um ${ }^{2}$ ) and the xylem area of small vascular bundle (SVXA, um²) (Additional file 8: Figure S6), were observed and measured using a microscopy (ZEISS AXIO, Germany). The average trait value of each accession was used in data analyses of GWAS.

\section{Genotyping}

The 27 K SNP genotype data of the 423 accessions was generated from the $3 \mathrm{~K} \mathrm{RGP}$ (Zheng et al. 2015). For those SNPs, only the alleles of highest frequency in the 423 panels were retained and other alleles of low frequency were considered missing. The heterozygous alleles were also eliminated. SNPs with missing rate over $20 \%$ and minor allele frequency (MAF) less than 5\% were removed. Finally, a total of 26,097 SNPs were used in the GWAS.

\section{Population structure and kinship}

Principal component (PC) and population structure (Q) were applied to infer population structure. For the 26,097 SNP, we further removed SNP loci with missing rate over $10 \%$ and MAF less than 0.10 . Then, 6601 evenly distributed SNPs with average marker spacing around $50 \mathrm{~kb}$ were sampled to calculate population structure (Q). A model based Bayesian clustering analysis method implemented in STRUCTURE software version 2.3.4 (Pritchard et al. 2000) was used. The program was run with the following parameters: $k$, the number of groups in the panel varying from 1 to $7 ; 10$ runs each $\mathrm{k}$ value; for each run, 10,000 burn in iterations followed by 10,000 MCMC (Markov Chain Monte Carlo) iterations. A principal component analysis (PCA) was performed the efficient mixed-model association (EMMA) method in the Genome Association and Prediction Integrated Tool (GAPIT) R package (Lipka et al. 2012) to examine the population structure. The K matrix (kinship matrix) was obtained from the results of the relatedness analysis using the EMMA method in GAPIT, a package of R software (Lipka et al. 2012). The PCA and $\mathrm{K}$ matrix were used in the following association analysis.

\section{GWAS and identification of candidate genes}

We performed a GWAS to excavate the SNPs significant associations with all measured traits using 26,097 SNPs and the mean trait values of the 423 accessions. In this study, the model of mixed linear (MLM), PCA $+\mathrm{K}$, was used in the association analysis. And we performed the study by the software GAPIT. The MLM includes both 
fixed and random effects. Including individuals as random effects gave an MLM the ability to incorporate information about relationships among individuals. This information about relationships was conveyed through the KI matrix, which was used in an MLM as the variancecovariance matrix between the individuals. Then a genetic marker-based KI matrix was used jointly with PCA (Yu et al. 2006). After the GWAS, SNPs affecting the measured traits were claimed when the test statistics reached $P<$ $1.0 \times 10^{-4}$ in at least one of the two years.

Gene-based association analysis was carried out to detect candidate genes for important QTL. Here, QTL regions meeting at least one of the following criteria were considered as important: (1) consistently identified in both two years; (2) affecting more than one trait; and/or (3) close to previously reported cloned genes or fine mapped QTL. The following five steps were conducted to identify candidate genes for important QTL identified. Firstly, the LD block was analyzed using the software "Haploview v4.2" (Barrett et al. 2005). A LD block was created when the upper 95\% confidence bounds of D' value exceeded 0.98 and the lower bounds exceeded 0.70 (Gabriel et al. 2002). The LD block where the significant trait-associated SNPs were situated was defined as the candidate gene regions. We can find all the genes located in the candidate regions for each important QTL from the Rice Annotation Project Database (RAPDB).Secondly, all available non-synonymous SNPs located inside of these genes were searched from $32 \mathrm{M}$ SNPs data generated from $3 \mathrm{~K}$ RGP in the Rice SNPSeek Database (Alexandrov et al. 2015). Thirdly, the SNPs with minor allele frequency less than 0.05 and/or missing rate over $20 \%$ were removed and remaining the high quality SNPs inside of these candidate genes of each important QTL were used to perform gene-based association analyses through MLM using the PCA and K applied in GWAS. The threshold was defined as $-\log _{10}$ $(P)$ of the peak SNP of the detected QTL minus 1 (Wang et al. 2017; Zhang et al. 2017). Fourthly, haplotype analysis were carried out for the candidate genes in each important QTL region using all non-synonymous SNPs located inside of the gene CDS region. Finally, candidate genes were determined by testing the significant differences among major haplotypes (containing more than 10 samples) for each important QTL through analysis of variance (ANOVA).

\section{Additional files}

Additional file 1: Figure S1. Distribution of SNP markers on chromosomes. The colors show the number of SNPs within $1 \mathrm{Mb}$ window size. (PPT $159 \mathrm{~kb}$ )

Additional file 2: Table S1. List of the 423 accessions including country of origin, subpopulation (indica, temperate japonica, tropical japonica, admixed japonica, admixed type, aus/boro, and basmati/sadri), population structure (Q), PC score, vascular bundle related traits measured in 2015 and 2016, and heading date measured in 2016. (XLSX 115 kb)

Additional file 3: Figure S2. Genome-wide association results for 8 vascular bundle related traits. Manhattan plots (left) and quantile-quantile plots (right) associated with LVN (a), LVA (b), LVPA (c), LVXA (d), SVN (e), SVA (f), SVPA (g), and SVXA (h) in 423 accessions in 2015 and 2016. For the Manhattan plots, $-\log _{10} P$-values from a genome-wide scan were plotted against the position of the SNPs on each of 12 chromosomes and the horizontal grey dashed lines show the suggestive threshold $\left(P=1.0 \times 10^{-4}\right)$. For the quantile-quantile plots, the horizontal axes indicate the $-\log _{10}$-transformed expected $P$ values, and the vertical axes indicate the $-\log _{10}$-transformed observed $P$-values. Arrows indicate QTL overlapping with the published QTL. (PPT 1286 kb)

Additional file 4: Figure S3. Comparisons of eight vascular bundle traits between indica and japonica subpopulations. Blue and orange bar indicate indica and japonica, respectively. All data are presented as the mean \pm SD. ${ }^{*}, P<0.05$ and ${ }^{* *}, P<0.001$. (PPT $190 \mathrm{~kb}$ )

Additional file 5: Table S2. Haplotype analysis of the targeted genes related to 9SVA8.1 and qSVXA8 in the whole population, indica and japonica subpopulations, respectively. The letters ( $a$, and b) indicate multiple comparison results at the significant level 0.05. (XLSX $10 \mathrm{~kb}$ )

Additional file 6: Figure S4. Comparison of LVA and LVPA between A and $\mathrm{G}$ alleles located in the third exon of Os04g0615000 (NAL1) gene. The letters on histogram ( $a$, and $b$ ) indicate multiple comparisons result at the significant level 0.05 . The value on the histogram is the number of individuals of each allele. (PPT $217 \mathrm{~kb}$ )

Additional file 7: Figure S5. Comparisons of heading date, grain number per panicle and plant height among different haplotypes of Ghd7.1 gene in 2016 Sanya. The letters on histogram (a, b and c) indicate multiple comparison results at the significant level 0.05 . The value on the histogram is the number of individuals of each haplotype. (PPT $218 \mathrm{~kb}$ )

Additional file 8: Figure S6. Cross-sections of large and small vascular bundle in peduncle stained with safranin $O$ staining. $P$, phloem; $X P$, xylem parenchyma; $P X$, protoxylem element; $M X$, metaxylem element; $P X L$, protoxylem lacuna. (PPT $699 \mathrm{~kb}$ )

\section{Abbreviations}

3 K RGP: 3 K Rice Genome Project; ANOVA: Analysis of variance; EMMA: Efficient mixed-model association; FAA: Formalin-acetic-alcohol; GAPIT: Genome association and prediction integrated tool; GWAS: Genomewide association study; Kl: Kinship; LD: Linkage disequilibrium; LVA: Total area of large vascular bundle; LVN: Number of large vascular bundle; LVPA: Phloem area of large vascular bundle; LVXA: Xylem area of large vascular bundle; MAF: Minor allele frequency; MAS: Marker-assisted selection; MLM: Model of mixed linear; PCA: Principal component analysis; PRB: Primary rachis branch; QTL: Quantitative trait loci/locus; RAP-DB: Rice annotation project database; SNP: Single-nucleotide polymorphism; SVA: Total area of small vascular bundle; SVN: Number of small vascular bundle; SVPA: Phloem area of small vascular bundle; SVXA: Xylem area of small vascular bundle

\section{Acknowledgements}

This work was funded by the National Natural Science Foundation of China (31671602), the Key Laboratory Project of Department of Education of Liaoning Province, China (LZ2015065), the national key research and development program of China (2016YFD0300104), the "863" Key Project (2014AA10A601) from the Chinese Ministry of Science and Technology and the Bill and Melinda Gates Foundation project (\#OPP1130530).

\section{Authors' contributions}

$Y W$, JLX and ZKL designed and supervised the research. $L Y Z, X Y W$, and $Y W$ performed phenotyping. LYZ, TQZ, and SW analyzed data. LYZ, YW and JLX wrote the paper. All authors read and approved the final manuscript.

\section{Competing interests}

The authors declare that they have no competing interests. 


\section{Publisher's Note}

Springer Nature remains neutral with regard to jurisdictional claims in published maps and institutional affiliations.

\section{Author details}

'Rice Research Institute, Shenyang Agricultural University/Key Laboratory of Northern Japonica Rice Genetics and Breeding, Ministry of Education, Shenyang 110866, China. ${ }^{2}$ Institute of Crop Sciences/National Key Facility for Crop Gene Resources and Genetic Improvement, Chinese Academy of Agricultural Sciences, 12\# South Zhong-Guan-Cun Street, Haidain District, Beijing 100081, China. ${ }^{3}$ Agricultrual Genomics Institute at Shenzhen, Chinese Academy of Agricultural Sceinces, Shenzhen 518120, China. ${ }^{4}$ Shenzhen Institute of Breeding and Innovation, Chinese Academy of Agricultural Sciences, Shenzhen 518120, China.

Received: 11 November 2017 Accepted: 12 February 2018 Published online: 06 March 2018

\section{References}

3K RGP (2014) The 3,000 rice genomes project. Gigascience 3:7

Alexandrov N, Tai S, Wang W, Mansueto L, Palis K, Fuentes RR, Ulat VJ, Chebotarov D, Zhang G, Li Z, Mauleon R, Hamilton RS, McNally KL (2015) SNP-seek database of SNPs derived from 3000 rice genomes. Nucleic Acids Res 43:1023-1027

Ashraf M, Akbar M, Salim M (1994) Genetic improvement in physiological traits of rice yield. In: Slafer GA (ed) Genetic improvement of field crops. Marcel Dekker Incorporates, New York, pp 413-455

Bai X, Wu B, Xing Y (2012) Yield-related QTLs and their applications in rice genetic improvement. J Integr Plant Biol 54:300-311

Barrett JC, Fry B, Maller J, Daly MJ (2005) Haploview: analysis and visualization of LD and haplotype maps. Bioinformatics 21:263-265

Berleth T, Scarpella E, Prusinkiewicz P (2007) Towards the systems biology of auxin-transport-mediated patterning. Trends Plant Sci 12:151-159

Cui K, Peng S, Xing Y, Yu S, Xu C, Zhang Q (2003) Molecular dissection of the genetic relationships of source, sink and transport tissue with yield traits in rice. Theor Appl Genet 106:649-658

Dettmer J, Elo A, Helariutta Y (2009) Hormone interactions during vascular development. Plant Mol Biol 69:347-360

Donald CM (1968) The breeding of crop ideotypes. Euphytica 17:385-403

Donner TJ, Sherr I, Scarpella E (2009) Regulation of preprocambial cell state acquisition by auxin signaling in Arabidopsis leaves. Development 136:3235-3246

Evans LT, Dunstone RL, Rawson HM, Williams RF (1970) The phloem of the wheat stem in relation to requirements for assimilate by the ear. Aust J Biol Sci 23: $743-752$

Fujita D, Trijatmiko KR, Tagle AG, Sapasap MV, Koide Y, Sasaki K, Tsakirpaloglou N, Gannaban RB, Nishimura T, Yanagihara S, Fukuta Y, Koshiba T, Slamet-Loedin IH, Ishimaru T, Kobayashi N (2013) Nal1 allele from a rice landrace greatly increases yield in modern indica cultivars. Proc Natl Acad Sci U S A 110 20431-20436

Fukushima A, Akita S (1997) Varietal differences of the course and differentiation time of large vascular bundles in the rachis of rice. Jpn J Crop Sci 66:24-28

Fukuyama T, Sasahara H, Fukuta Y (1996) New characters for identification of indica and japonica rice. Rice Genet Newsl 13:48-50

Fukuyama T, Takayama T (1995) Variations of the vascular bundle system in Asian rice cultivars. Euphytica 86:227-231

Gabriel SB, Schaffner SF, Nguyen H, Moore JM, Roy J, Blumenstiel B, Higgins J, DeFelice M, Lochner A, Faggart M, Liu-Cordero SN, Rotimi C, Adeyemo A, Cooper R, Ward R, Lander ES, Daly MJ, Altshuler D (2002) The structure of haplotype blocks in the human genome. Science 296:2225-2229

Gao H, Jin M, Zheng XM, Chen J, Yuan D, Xin Y, Wang M, Huang D, Zhang Z, Zhou K, Sheng P, Ma J, Ma W, Deng H, Jiang L, Liu S, Wang H, Wu C, Yuan L, Wan J (2014) Days to heading 7, a major quantitative locus determining photoperiod sensitivity and regional adaptation in rice. Proc Natl Acad Sci U S A 111:16337-16342

Han B, Huang X (2013) Sequencing-based genome-wide association study in rice. Curr Opin Plant Biol 16:133-138

Housley TL, Peterson DM (1982) Oat stem vascular size in relation to kernel number and weight. II. Field Environment1. Crop Sci 22:274-278

Huang J, Li Z, Zhao D (2016) Deregulation of the OsmiR160 target gene OsARF18 causes growth and developmental defects with an alteration of auxin signaling in rice. Sci Rep 6:29938
Huang X, Wei X, Sang T, Zhao Q, Feng Q, Zhao Y, Li C, Zhu C, Lu T, Zhang Z, Li M, Fan D, Guo Y, Wang A, Wang L, Deng L, Li W, Lu Y, Weng Q, Liu K, Huang T, Zhou T, Jing Y, Li W, Lin Z, Buckler ES, Qian Q, Zhang QF, Li J, Han B (2010) Genome-wide association studies of 14 agronomic traits in rice landraces. Nat Genet 42:961-967

Lafitte HR, Travis RL (1984) Photosynthesis and assimilate partitioning in closely related lines of rice exhibiting different sink: source relationships. Crop Sci 24 447-452

Lipka AE, Tian F, Wang Q, Peiffer J, Li M, Bradbury PJ, Gore MA, Buckler ES, Zhang Z (2012) GAPIT: genome association and prediction integrated tool. Bioinformatics 28:2397-2399

Liu T, Liu H, Zhang H, Xing Y (2013) Validation and characterization of Ghd7.1, a major QTL with pleiotropic effects on spikelets per panicle, plant height and heading date in rice (Oryza sativa L.) J Integr Plant Biol 55:917-927

Liu X, Wei X, Sheng Z, Jiao G, Tang S, Luo J, Hu P (2016) Polycomb protein OsFIE2 affects plant height and grain yield in rice. PLoS One 11:e0164748

Lucas WJ, Groover A, Lichtenberger R, Furuta K, Yadav SR, Helariutta Y, He XQ, Fukuda H, Kang J, Brady SM, Patrick JW, Sperry J, Yoshida A, López-Millán AF, Grusak MA, Kachroo P (2013) The plant vascular system: evolution, development and functions. J Integr Plant Biol 55:294-388

Ma L, Sang X, Zhang T, Yu Z, Li Y, Zhao F, Wang Z, Wang Y, Yu P, Wang N, Zhang C, Ling Y, Yang Z, He G (2017) ABNORMAL VASCULAR BUNDLES regulates cell proliferation and procambium cell establishment during aerial organ development in rice. New Phytol 213:275-286

Mattsson J, Ckurshumova W, Berleth T (2003) Auxin signaling in arabidopsis leaf vascular development. Plant Physiol 131:1327-1339

Morishima H, Oka HI (1981) Phylogenetic differentiation of cultivated rice, XXII. Numerical evaluation of the Indica-Japonica differentiation. Jpn J Breed 31: 402-413

Nagato K, Chaudhry FM (1969) A comparative study of ripening process and kernel development in japonica and indica rice. Proc Crop Sci Soc Japan 38 $425-433$

Osada A, Ishizaki Y, Suzuki S (1983) Difference in the number of days for ripening of grains between japonica and indica rice varieties. Japan J. Trop Agric 27: $59-66$

Paciorek T, Zazimalova E, Ruthardt N, Petrasek J, Stierhof YD, Kleine-Vehn J, Morris DA, Emans N, Jurgens G, Geldner N, Friml J (2005) Auxin inhibits endocytosis and promotes its own efflux from cells. Nature 435:1251-1256

Pritchard JK, Stephens M, Donnelly P (2000) Inference of population structure using multilocus genotype data. Genetics 155:945-959

Qi J, Qian Q, Bu Q, Li S, Chen Q, Sun J, Liang W, Zhou Y, Chu C, Li X, Ren F, Palme K, Zhao B, Chen J, Chen M, Li C (2008) Mutation of the rice narrow leaf1 gene, which encodes a novel protein, affects vein patterning and polar auxin transport. Plant Physiol 147:1947-1959

Sachs T (1981) The control of the patterned differentiation of vascular tissues. Adv Bot Res 9:151-262

Sasahara H, Fukuta Y, Fukuyama T (1999) Mapping of QTLs for vascular bundle system and spike morphology in rice, Oryza sativa L. Breed Sci 49:75-81

Scarpella E, Marcos D, Friml J, Berleth T (2006) Control of leaf vascular patterning by polar auxin transport. Dev Biol 20:1015-1027

Sun C, Hu Z, Zheng T, Lu K, Zhao Y, Wang W, Shi J, Wang C, Lu J, Zhang D, Li Z, Wei C (2017) RPAN: rice pan-genome browser for 3000 rice genomes. Nucleic Acids Res 45:597-605

Taguchi-Shiobara F, Ota T, Ebana K, Ookawa T, Yamasaki M, Tanabata T, Yamanouchi U, Wu J, Ono N, Nonoue Y, Nagata K, Fukuoka S, Hirabayashi H, Yamamoto T, Yano M (2015) Natural variation in the flag leaf morphology of rice due to a mutation of the narrow leaf1 gene in Onyza sativa L. Genetics 201:795-808

Takai T, Adachi S, Taguchi-Shiobara F, Sanoh-Arai Y, Iwasawa N, Yoshinaga S, Hirose S, Taniguchi Y, Yamanouchi U, Wu J, Matsumoto M, Sugimoto K, Kondo K, Ikka T, Ando T, Kono I, Ito S, Shomura A, Ookawa T, Hirasawa T, Yano M, Kondo M, Yamamoto T (2013) A natural variant of NAL1, selected in high-yield rice breeding programs, pleiotropically increases photosynthesis rate. Sci Rep 3:2149

Terao T, Nagata K, Morino K, Hirose T (2010) A gene controlling the number of primary rachis branches also controls the vascular bundle formation and hence is responsible to increase the harvest index and grain yield in rice. Theor Appl Genet 120:875-893

Wang XQ, Pang YL, Wang CC, Chen K, Zhu YJ, Shen C, Ali J, Xu JL, Li ZK (2017) New candidate genes affecting rice grain appearance and milling quality detected by genome-wide and gene-based association analyses. Front Plant Sci 7:1998 
Weng JH, Chen CY (1987) Differences between indica and japonica rice varieties in $\mathrm{CO} 2$ exchange rates in response to leaf nitrogen and temperature. Photosynth Res 14:171-178

Xu JL, Wang Y, Zhang F, Wu Y, Zheng TQ, Wang YH, Zhao XQ, Cui YR, Chen K, Zhang Q, Lin HX, Li JY, Li ZK (2015) SS1 (NAL1)- and SS2-mediated genetic networks underlying source-sink and yield traits in rice (Oryza sativa L.) PLoS One 10:e0132060

Yano K, Yamamoto E, Aya K, Takeuchi H, Lo PC, Hu L, Yamasaki M, Yoshida S, Kitano H, Hirano K, Matsuoka M (2016) Genome-wide association study using whole-genome sequencing rapidly identifies new genes influencing agronomic traits in rice. Nat Genet 48:927-934

Yu J, Pressoir G, Briggs WH, Vroh Bi I, Yamasaki M, Doebley JF, McMullen MD, Gaut BS, Nielsen DM, Holland JB, Kresovich S, Buckler ES (2006) A unified mixed-model method for association mapping that accounts for multiple levels of relatedness. Nat Genet 38:203-208

Zhang J, Chen K, Pang YL, Naveed SA, Zhao XQ, Wang XQ, Wang Y, Dingkuhn M, Pasuquin J, Li ZK, Xu JL (2017) QTL mapping and candidate gene analysis of ferrous iron and zinc toxicity tolerance at seedling stage in rice by genomewide association study. BMC Genomics 18:828

Zhang ZH, Li P, Wang LX, Tan CJ, Hu ZL, Zhu YG, Zhu LH (2002) Identification of quantitative trait loci (QTLs) for the characters of vascular bundles in peduncle related to indica-japonica differentiation in rice (Oryza sativa L.) Euphytica 128:279-284

Zhao K, Tung CW, Eizenga GC, Wright MH, Ali ML, Price AH, Norton GJ, Islam MR, Reynolds A, Mezey J, McClung AM, Bustamante CD, McCouch SR (2011) Genome-wide association mapping reveals a rich genetic architecture of complex traits in Oryza sativa. Nat Commun 2:467

Zheng TQ, Yu H, Zhang HL, Wu ZC, Wang WS, Tai SS, Chi L, Ruan J, Wei CC, Shi JX, Gao YM, Fu BY, Zhou YL, Zhao XQ, Zhang F, Mcnally KL, Li ZC, Zhang GY, Li JY, Zhang DB, Xu JL, Li ZK (2015) Rice functional genomics and breeding database (RFGB): 3K rice SNP and InDel sub-database (in Chinese). Chin Sci Bull 60:367-371

\section{Submit your manuscript to a SpringerOpen ${ }^{\circ}$ journal and benefit from:}

- Convenient online submission

- Rigorous peer review

- Open access: articles freely available online

- High visibility within the field

- Retaining the copyright to your article

Submit your next manuscript at springeropen.com 